\title{
Automated data Mining of the electronic health record for investigation of healthcare-associated outbreaks — CORRIGENDUM
}

In the original published article by Sundermann et $\mathrm{al}^{1}$, the affiliation superscript for author Jieshi Chen was incorrect. The correct superscript for “Auton Laboratory, Carnegie Mellon University, Pittsburgh, Pennsylvania” has been added to Jieshi Chen's name in the byline. The publisher apologizes for the error.

\section{Reference}

1. Sundermann, AJ, Miller, JK, Marsh, JW, et al. Automated data mining of the electronic health record for investigation of healthcare-associated outbreaks. Infection Control Hosp Epidemiol 2019;40:314-319. 